\title{
Clinical observation on the application of transcatheter arterial chemoembolization combined with radiofrequency ablation in the treatment of large hepatocellular carcinoma.
}

\author{
Feng Zheng ${ }^{1}$, Ji-Shu Quan ${ }^{2}$, Xiang-Dan Cui ${ }^{3}$, Xue-Feng $\mathrm{Jin}^{3}$, Xue-Zhe Yin ${ }^{3 *}$ \\ ${ }^{1}$ Department of Oncology, Linyi People's Hospital, Linyi, Shandong, PR China \\ ${ }^{2}$ Department of Medicine, Yanbian University, Yanji, Jilin, PR China \\ ${ }^{3}$ Department of Respiratory Medicine, Affiliated Hospital of Yanbian University, Yanji, Jilin, PR China
}

\begin{abstract}
Objective: This study aimed to determine the clinical effect of arterial chemoembolization transcatheter (TACE) combined with radiofrequency ablation during the clinical treatment of large hepatocellular carcinoma.

Methods: A total of 82 patients with large hepatocellular carcinoma were selected as experimental subjects. These patients received treatment at our hospital from May 2015 to January 2017. Digital parity method was used to divide patients with large hepatocellular carcinoma into two groups. For the control group (41 patients), TACE was adopted for the clinical treatment of large hepatocellular carcinoma. For the observation group (41 patients), TACE combined with Radiofrequency Ablation (RFA) was utilized. Finally, the two groups of patients with large hepatocellular carcinoma were compared in terms of disease treatment effect, treatment satisfaction, liver-function-related indicator, and immune function.

Results: The total effective rate of patients with large hepatocellular carcinoma in the observation group (97.56\%) exhibited significant improvement compared with that of patients in the control group $(65.85 \%)(P<0.05)$. The treatment satisfaction of patients in the observation group $(97.56 \%)$ was significantly higher than that of patients in the control group $(68.29 \%)(P<0.05)$. Furthermore, the liverfunction-related index of patients in the observation group displayed significant improvement compared with that of patients in the control group $(P<0.05)$. The immune function of patients in the observation group also showed significant improvement compared with that of patients in the control group (P<0.05).

Conclusion: During the clinical treatment of large hepatocellular carcinoma, TACE combined with RFA for clinical treatment can improve the treatment efficacy, immune function, and liver function index of patients, thereby improving the prognostic ability of patients with large hepatocellular carcinoma.
\end{abstract}

Keywords: Large hepatocellular carcinoma, Transcatheter arterial chemoembolization (TACE), Radiofrequency ablation, Clinical effect.

Accepted on November 16, 2017

\section{Introduction}

Hepatocellular carcinoma is one of the most common types of malignant liver tumors [1]. This type is characterized with insidious and slow onset of disease and significant mortality. Clinical treatment is significantly difficult in patients with large hepatocellular carcinoma who missed the opportunity of surgical operation [2,3]. Currently, Transcatheter Arterial Chemoembolization (TACE) is widely adopted in the treatment of patients with large hepatocellular carcinoma. However, the TACE method cannot exert a significant curative effect on patients with occult ectopic arteries, portal vein blood supply, or portal cancer embolus [4]. On the contrary, the patients show a significant recurrence rate with this method. Patients are commonly treated by clinical percutaneous Radiofrequency Ablation (RFA), which is characterized with minor trauma to patients, convenient operation, and low incidence rate of complications. The current research aims to determine an effective method to treat patients with large hepatocellular carcinoma and the clinical application value of TACE combined with RFA. 


\section{Data and Methods}

\section{General information}

A total of 82 patients with large hepatocellular carcinoma were selected as experimental subjects. These patients received treatment at our hospital from May 2015 to January 2017. Digital parity method was used to divide all these patients with large hepatocellular carcinoma into two groups. The control group (41 patients) comprised 22 male patients and 19 female patients; the age range is $46-79$, and the average age is $58.63 \pm$ 4.22; the patients' lesion diameter is in the range of $6-14 \mathrm{~cm}$, and the average lesion diameter is $6.33 \pm 1.25 \mathrm{~cm}$. The observation group (41 patients) comprised 25 male patients and 16 female patients; the age range is $49-81$, and the average age is $58.65 \pm 4.26$; the patients' lesion diameter is in the range of $6-15 \mathrm{~cm}$, and the average lesion diameter is $6.35 \pm 1.29 \mathrm{~cm}$. The two patient groups showed no significant difference in terms of gender, age, and lesion diameter $(\mathrm{P}>0.05)$.

\section{Selection criteria}

Pathological findings were confirmed clinically for patients with hepatocellular carcinoma who were not suitable for or disagreed with surgical resection. The inclusion criteria were as follows: patients showed tumors within the liver only and no extrahepatic metastasis. The tumor diameter was larger than 5 $\mathrm{cm}$ but less than $10 \mathrm{~cm}$. The patients' hepatic veins or portal veins showed no tumor emboli. The liver function Child-Pugh grade of patients was A or B.

\section{Methods}

Control group method: TACE was adopted in the clinical treatment of large hepatocellular carcinoma. Prior to the treatment, all of the patients were systematically examined. Patients with no contraindication were treated by clinic surgery. Prior to the operation, the patients were asked to stop eating excitant food. In addition, patients were asked to drink no water and eat none for $4 \mathrm{~h}$ before the surgery. Routine disinfection and towel laying procedures were completed according to the protocol [5]. Afterward, general anesthesia was administered. Patients were treated by clinical femoral artery puncture and subsequent mesenteric and hepatic artery angiographies to further understand the location and size of tumor and blood supply condition. A catheter was inserted through the right femoral artery of the patients. A mixture of pharmorubicin (20 mg), cis-platinum complexes (80 mg), idolized oil $(15 \mathrm{ml})$, and pirarubicin $(30 \mathrm{mg})$ was prepared for chemotherapy operation. After drug injection, accessory embolization was implemented with a gelatin sponge at the end of the patients' artery. After the surgery, the patients' health conditions were observed to prevent patients from having liver rupture symptoms, prepare for emergency, provide necessary guidance for comfort, help patients choose between the semireclining and supine positions, provide rational local massage on patients, and significantly reduce the patients' pain.
Observation group method: In the clinical treatment of large hepatocellular carcinoma, the TACE method combined with RFA was adopted. The specific steps of TACE are the same with those in the control group. After $2 \mathrm{w}$ of chemoembolization, patients were treated by RFA. Specifically, patients were treated with intramuscular injection of pethidine (75 mg) before operation. The American RADION-ICS cold circulation RF system was mainly used in treating patients. After administering local anesthesia clinically, CT guidance was used to apply puncture operation on patients until the contralateral edge of tumor was reached [6,7]. Physiological saline was prepared for the perfusion of patients, and a reasonable constant temperature cold circulation treatment was carried out. During the treatment process, the treatment frequency was $40 \mathrm{~W}$, and the treatment time was 15 min. The RFA time was reasonably increased or decreased according to the lesion location, lesion diameter, and patient precipitation. Under normal circumstances, the lesion diameter is within $5-10 \mathrm{~cm}$, and the treatment duration is $30-60 \mathrm{~min}$. After guaranteeing the interval between $1 \mathrm{w}$ and $2 \mathrm{w}$, the patients were provided with clinical treatment again. After completing the treatment, reasonable hemostatic treatment was also carried out. Antibiotic drugs were also prepared for infection prevention according to the doctor's advice.

\section{Evaluation criteria}

Criteria for evaluation efficacy: Completely relieved: The tumor symptom of patients with large hepatocellular carcinoma completely disappears after receiving treatment. Partially relieved: the tumor size of patients with large hepatocellular carcinoma is reduced by half after receiving treatment, and no new lesion is observed. No change: After receiving treatment, the tumor volume of patients decreases by $25 \%-50 \%$. Progressive: After receiving treatment, the tumor volume increases by more than $25 \%$.

Evaluation criteria for treatment satisfaction: The questionnaire survey developed by our hospital was used to investigate the treatment satisfaction of all patients with large hepatocellular carcinoma. Evaluation was conducted according to three aspects: satisfactory treatment, barely satisfactory treatment, and unsatisfactory treatment.

\section{Statistical methods}

Statistical software SPSS20.0 was used for the statistical analysis of the treatment results of all patients with large hepatocellular carcinoma. The count data (treatment effect) of the two groups were compared using the $\%$ form to complete the $\chi^{2}$ test. The measurement data (immune function index) of the two groups were compared using the form $\overline{\mathrm{x}} \pm \mathrm{s}$ to complete the t-test. $\mathrm{P}<0.05$ was considered to indicate significant difference and statistical significance. 
Clinical observation on the application of transcatheter arterial chemoembolization combined with radiofrequency ablation in the treatment of large hepatocellular carcinoma

\section{Results}

\section{Comparison of clinical treatment effect}

The total effective rate of patients with large hepatocellular carcinoma in the observation group (97.56\%) significantly increased compared with that of patients in the control group $(65.85 \%)(\mathrm{P}<0.05)($ Table 1$)$.

Table 1. Clinical comparison of the total effective rate of patients with large hepatocellular carcinoma in two groups (patients).

\begin{tabular}{|c|c|c|c|c|c|c|}
\hline Group & $\mathbf{n}$ & $\begin{array}{l}\text { Completely } \\
\text { relieved }\end{array}$ & $\begin{array}{l}\text { Partially } \\
\text { relieved }\end{array}$ & $\begin{array}{l}\text { No } \\
\text { change }\end{array}$ & $\begin{array}{l}\text { Progressiv } \\
\text { e }\end{array}$ & $\begin{array}{l}\text { Total } \\
\text { effectiv }\end{array}$ \\
\hline
\end{tabular}

\begin{tabular}{llllllll} 
& & & & & $\begin{array}{l}\text { e rate } \\
\text { (\%) }\end{array}$ \\
\hline $\begin{array}{l}\text { Observatio } \\
\mathrm{n}\end{array}$ & 41 & 35 & 5 & 1 & 0 & 97.56 \\
\hline Control & 41 & 21 & 6 & 12 & 2 & 65.85 \\
\hline $\mathrm{X}^{2}$ & & & & & & 13.7891 \\
\hline $\mathrm{P}$ & & & & & & & $<0.05$ \\
\hline
\end{tabular}

\section{Comparison of treatment satisfaction}

The treatment satisfaction of patients with large hepatocellular carcinoma in the observation group $(97.56 \%)$ showed a significant increase compared with that of patients in the control group (68.29\%) $(\mathrm{P}<0.05)$ (Table 2).

Table 2. Clinical comparison of treatment satisfaction of patients with large hepatocellular carcinoma in two groups (patients).

\begin{tabular}{|c|c|c|c|c|c|c|c|c|}
\hline Group & $\mathbf{n}$ & Satisfactory & \multicolumn{2}{|c|}{ Barely satisfactory } & Unsatisfactory & & \multicolumn{2}{|c|}{ Total satisfaction (\%) } \\
\hline Observation Group & 41 & 31 & 9 & & 1 & & 97.56 & \\
\hline Control Group & 41 & 17 & 11 & & 13 & & 68.29 & \\
\hline$x^{2}$ & & & & & & & 12.4034 & \\
\hline$P$ & & & & & & & $<0.05$ & \\
\hline \multicolumn{4}{|c|}{ Comparison of liver function index } & $\mathrm{t}$ & 3.3258 & 10.5251 & 17.5173 & 9.7733 \\
\hline \multicolumn{4}{|c|}{ The liver function index of patients with large hepatocellular } & $P$ & $<0.05$ & $<0.05$ & $<0.05$ & $<0.05$ \\
\hline
\end{tabular}
carcinoma in the observation group displayed significant improvement compared with that of patients in the control group $(\mathrm{P}<0.05)$ (Table 3$)$.

Table 3. Clinical comparison of liver function index of patients with large hepatocellular carcinoma in two groups $(\bar{x} \pm s)$.

\begin{tabular}{lllll}
\hline Groups & $\mathbf{n}$ & TBIL $(\mu \mathrm{mol} / \mathrm{L})$ & ALT $(\mathbf{u} / \mathbf{L})$ & AFP $(\mathbf{n g} / \mathbf{m l})$ \\
\hline $\begin{array}{l}\text { Observation } \\
\text { Group }\end{array}$ & 41 & $25.76 \pm 12.25$ & $53.85 \pm 18.50$ & $77.62 \pm 39.30$ \\
\hline Control Group & 41 & $37.13 \pm 11.90$ & $42.15 \pm 18.29$ & $135.22 \pm 42.50$ \\
\hline $\mathrm{t}$ & 4.2628 & 2.8797 & 6.3715 \\
\hline $\mathrm{P}$ & $<0.05$ & $<0.05$ & $<0.05$ \\
\hline
\end{tabular}

\section{Comparison of immune function index}

The immune function index of patients with large hepatocellular carcinoma in the observation group showed significant improvement compared with that of patients in the control group $(\mathrm{P}<0.05)$ (Table 4).

Table 4. Clinical comparison of immune function index of patients with large hepatocellular carcinoma in two groups $(\bar{x} \pm s)$.

\begin{tabular}{llllll}
\hline Groups & $\mathbf{n}$ & $\mathbf{C D}^{+}(\%)$ & $\mathbf{C D 4}^{+}(\%)$ & CD8 $^{+}(\%)$ & CD4 $^{+} / \mathbf{C D}^{+}$ \\
\hline Observation & 41 & $48.39 \pm 8.49$ & $39.43 \pm 5.10$ & $21.45 \pm 3.50$ & $1.75 \pm 0.46$ \\
\hline Control & 41 & $42.53 \pm 7.43$ & $27.85 \pm 4.86$ & $35.03 \pm 3.52$ & $0.92 \pm 0.29$
\end{tabular}

\section{Discussion}

When providing treatment for patients with large hepatocellular carcinoma, TACE combined with RFA can exert significant effect. In the process hepatic artery embolization, the common hepatic artery of patients is mainly selected for treatment [8]. The RF electrode can effectively enter the tumor through imaging guidance. Moreover, the thermal efficiency function of RFA can significantly promote the necrosis of tissues surrounding the tumor so as to remove tumor completely [9]. This method poses small trauma to patients. RFA is also characterized with convenient operation; it can also significantly slow the blood supply and blood flow rate of tumor in patients and significantly reduce tumor temperature. In addition, embolization agent and idolized oil are used to block the portal vein around the tumor during chemotherapy [10]. Thus, the treatment range of radiofrequency ablation is significantly increased.

Result analysis showed that patients with large hepatocellular carcinoma in the observation group who were treated with TACE combined with RFA showed better treatment effect, treatment satisfaction, liver function index, and immune function index than those of patients in the control group who received TACE treatment alone. The disease was successfully relieved in the observation group, thereby obtaining accurate treatment effect and highlighting the combined clinical value of TACE and RFA. 


\section{Conclusion}

In the clinical treatment of large hepatocellular carcinoma, TACE combined with RFA can improve the treatment effect, treatment satisfaction, liver-function-related index, and immune function index of patients. Consequently, the prognostic and living abilities of patients with large hepatocellular carcinoma are improved.

\section{References}

1. Kakar S, Grenert JP, Paradis V, Pote N, Jakate S, Ferrell LD. Hepatocellular carcinoma arising in adenoma: similar immunohistochemical and cytogenetic features in adenoma and hepatocellular carcinoma portions of the tumor. Mod Pathol 2014; 27: 1499-1509.

2. Cillo U, Giuliani T, Polacco M, Manley LMH, Crivellari G, Vitale A. Prediction of hepatocellular carcinoma biological behavior in patient selection for liver transplantation. World J Gastroenterol 2016; 22: 232-252.

3. Niu ZS1, Niu XJ1, Wang WH1. Genetic alterations in hepatocellular carcinoma: An update. World J Gastroenterol 2016; 22: 9069-9095.

4. Chung JW, Kim HC, Yoon JH, Lee HS, Jae HJ, Lee W, Park JH. Transcatheter arterial chemoembolization of hepatocellular carcinoma: prevalence and causative factors of extrahepatic collateral arteries in 479 patients. Korean $\mathrm{J}$ Radiol 2006; 7: 257-266.

5. Suh CH, Shin JH, Yoon HM, Yoon HK, Ko GY, Gwon DI, Kim JH, Sung KB. Angiographic evaluation of hepatic arterial injury after cisplatin and Gelfoam-based transcatheter arterial chemoembolization for hepatocellular carcinoma in a 205 patient cohort during a 6-year followup. Br J Radiol 2014; 87: 20140054.
6. Zheng J, Sun B, Liu D, Yan L, Wang Y. Treatment with transcatheter arterial chemoembolization induces an increase of the L-selectinlow CXCR3 + CD8 + T cell subset in patients with hepatocellular carcinoma. Onco Targets Ther 2012; 5: 103-109.

7. Ahmed M, Kumar G, Moussa M, Wang Y, Rozenblum N, Galun E, Goldberg SN. Hepatic radiofrequency ablationinduced stimulation of distant tumor growth is suppressed by c-met inhibition. Radiology 2016; 279: 103-117.

8. Aras D, Topaloglu S, Cay S, Ozeke O, Ozcan F, Cagirci G. Pulmonary vein isolation using multi-electrode radiofrequency vs. conventional point-by-point radiofrequency ablation: A meta-analysis of randomized and non-randomized studies. Indian Pacing Electrophysiol J 2017; 17: 36-43.

9. Li C, Jia L, Wang Z, Niu L, An X. The efficacy of radiofrequency ablation in the treatment of pediatric arrhythmia and its effects on serum IL-6 and hs-CRP. Exp Ther Med 2017; 14: 3563-3568.

10. He ZX, Xiang P, Gong JP, Cheng NS, Zhang W. Radiofrequency ablation versus resection for Barcelona clinic liver cancer very early/early stage hepatocellular carcinoma: a systematic review. Ther Clin Risk Manag 2016; 12: 295-303.

\section{*Correspondence to}

Xue-Zhe Yin

Department of Respiratory Medicine

Affiliated Hospital of Yanbian University

PR China 